\title{
THE SPITZER SURVEYS OF THE SMALL MAGELLANIC CLOUD: INSIGHTS INTO THE LIFE-CYCLE OF POLYCYCLIC AROMATIC HYDROCARBONS
}

\author{
K.M. Sandstrom ${ }^{1}$, A.D. Bolatto ${ }^{2}$, B.T. Draine ${ }^{3}$, C. Bot ${ }^{4}$ and S. \\ Stanimirovic $^{5}$
}

\begin{abstract}
We present the results of two studies investigating the abundance and physical state of polycyclic aromatic hydrocarbons (PAHs) in the Small Magellanic Cloud (SMC). Observations with ISO and Spitzer have shown that PAHs are deficient in low-metallicity galaxies. In particular, galaxies with $12+\log (\mathrm{O} / \mathrm{H})<8$ show mid-IR SEDs and spectra consistent with low PAH abundance. The SMC provides a unique opportunity to map the PAH emission in a low-metallicity (12 $+\log (\mathrm{O} / \mathrm{H}) \sim 8)$ galaxy at high spatial resolution and sensitivity to learn about the PAH life cycle. Using mid- and far-IR photometry from the Spitzer Survey of the SMC $\left(S^{3} \mathrm{MC}\right)$ and mid-IR spectral mapping from the Spitzer Spectroscopic Survey of the SMC ( $\left.\mathrm{S}^{4} \mathrm{MC}\right)$ we determine the PAH abundance across the galaxy. We find that the SMC $\mathrm{PAH}$ abundance is low compared to the Milky Way and variable, with high abundance in molecular regions and low abundance in the diffuse ISM. From the variations of the mid-IR band strengths, we show that PAHs in the SMC are smaller and more neutral than their counterparts in more metal-rich galaxies. Based on the results of these two studies we propose that PAHs in the SMC are formed with a size distribution shifted towards smaller grains and are therefore easier to destroy under typical diffuse ISM conditions. The distribution of PAH abundance in the SMC suggests that PAH formation in molecular clouds is an important process. We discuss the implications of these results for our understanding of the PAH life-cycle both at low-metallicity and in the Milky Way.
\end{abstract}

\footnotetext{
1 Max-Planck-Institute for Astronomy, Konigstuhl 17, 69117 Heidelberg, Germany

2 Department of Astronomy and Laboratory for Millimeter-Wave Astronomy, University of Maryland, College Park, MD, USA

3 Department of Astrophysical Sciences, Princeton University, NJ, USA

4 Observatoire Astronomiques de Strasbourg, Université Louis Pasteur, 67000 Strasbourg, France

${ }^{5}$ Department of Astronomy, University of Wisconsin, Madison, WI, USA
} 


\section{Introduction}

Polycyclic aromatic hydrocarbons (PAHs) are a crucial component of interstellar dust. In addition to being responsible for a significant fraction of the total infrared emission of a galaxy, they can be a dominant source of photoelectrons and participate in chemical reactions across a variety of ISM phases. We would like to understand how the amount and characteristics of PAHs vary with galaxy type, star-formation history and ISM environment. All of these issues are tied to the life cycle of PAHs-the balance between their formation, destruction and processing in the ISM. PAH formation is thought to occur in the atmosphere of evolved stars (Latter 1991; Cherchneff et al. 1992), although it is not clear that evolved stars alone can account for the abundance of PAHs we observe in the ISM (Matsuura et al. 2009). It may also be the case that PAH formation in the ISM can be a significant source of PAHs (for a recent review, see Draine 2009). PAHs are observed to be destroyed in ionized gas in H II regions. Shock waves have been argued to be either a source (via grain shattering; Miville-Deschenes et al. 2002) or sink (Micelotta et al. 2010) of PAHs.

Although many aspects of the life cycle of PAHs are still poorly understood, we do know that it changes dramatically as a function of metallicity (see contributions by Calzetti et al. and Hunt et al. in this Volume). Observations with ISO and Spitzer have demonstrated that low metallicity galaxies have a deficit of PAHs relative to the total amount of dust (e.g. Engelbracht et al. 2005). Draine et al. (2007) used SED modeling to determine the PAH fraction $q_{\mathrm{PAH}}$ (defined as the fraction of the dust mass contributed by PAHs with sizes less than $10^{3}$ carbon atoms) in the SINGS galaxies. They found that at a metallicity of $12+\log (\mathrm{O} / \mathrm{H}) \sim$ 8 , galaxies transition from having a median $q_{\mathrm{PAH}} \sim 3.5 \%$, similar to the Milky Way value of $4.6 \%$, to a median of $\sim 1 \%$. Spectroscopic (Wu et al. 2006; Engelbracht et al. 2008) and photometric investigations (Marble et al. 2010) of large samples of low-metallicity galaxies provide clear evidence for this deficit.

The cause of the low-metallicity PAH deficit has been the subject of much investigation. Galliano et al. (2008) suggest that the deficit is related to PAH production due to the time delay between supernova and evolved star enrichment of the ISM. Others have argued that the deficit is due to destruction of PAHs by harder and more intense UV fields (Madden 2000; Gordon et al. 2008) or enhanced supernova shock destruction (O'Halloran et al. 2006). The Local Group galaxies, particularly the Small Magellanic Cloud, provide a test case for these scenarios. With a distance of $\sim 60 \mathrm{kpc}$ and a metallicity of $12+\log (\mathrm{O} / \mathrm{H}) \sim 8$, the SMC is a location where we can study PAHs at high spatial resolution and sensitivity in a galaxy right at the metallicity of the transition to PAH deficiency.

We have performed two surveys of the SMC using the Spitzer Space Telescope. The first, the Spitzer Survey of the SMC $\left(\mathrm{S}^{3} \mathrm{MC}\right)$, imaged the main star-forming areas of the Wing and Bar of the galaxy using all of the photometric bands of IRAC and MIPS. Details of $\mathrm{S}^{3} \mathrm{MC}$ and the data processing can be found in Bolatto et al. (2007). The second survey, the Spitzer Spectroscopic Survey of the SMC ( $\left.\mathrm{S}^{4} \mathrm{MC}\right)$, consisted of extensive spectral mapping observations using the low 


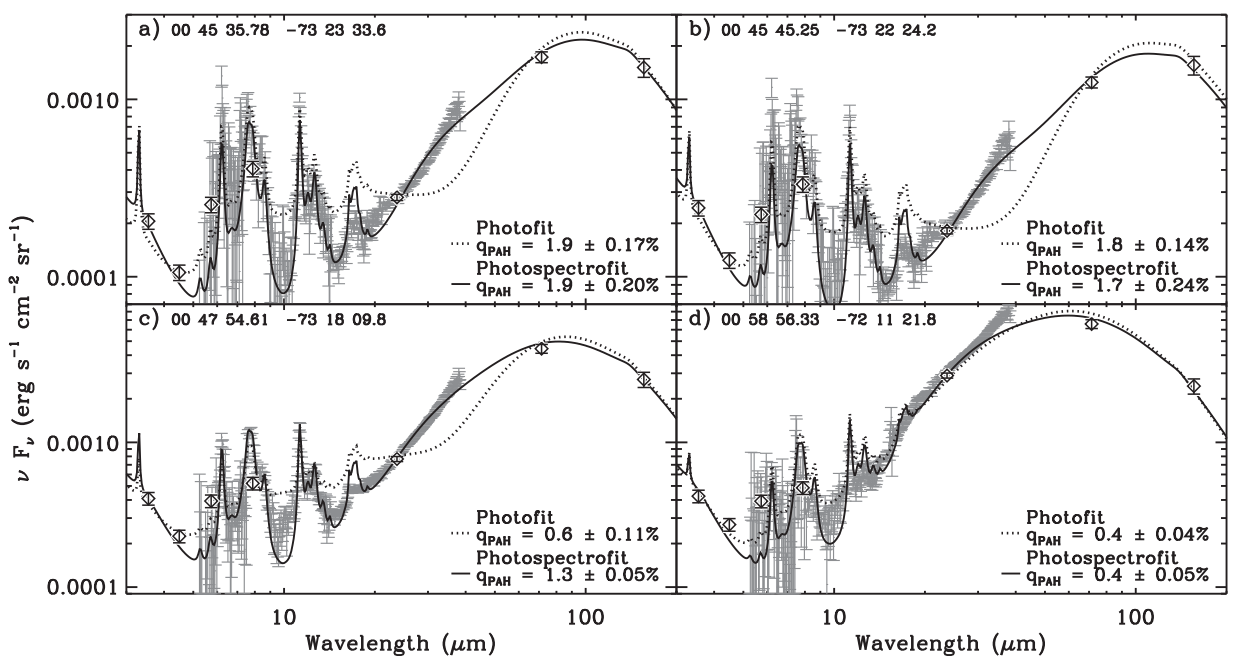

Fig. 1. Best fit models for photometry alone (photofit) and photometry and spectroscopy combined (photospectrofit) for a few selected regions covered by $\mathrm{S}^{3} \mathrm{MC}$ and $\mathrm{S}^{4} \mathrm{MC}$.

resolution modules $(5-35 \mu \mathrm{m})$ of IRS covering six of the main star-forming regions in the galaxy. The details of the survey and the data reduction can be found in Sandstrom et al. (2009) and Sandstrom et al. (2010, in prep.).

\section{The PAH fraction in the SMC}

In order to understand the cause of the PAH deficit in low-metallicity galaxies, we would like to answer the following questions about the SMC: 1) is the SMC deficient in PAHs, 2) are there spatial variations in the PAH fraction in the SMC, and 3) if there are spatial variations, with what are they correlated? To answer these questions we have made a map of the PAH fraction $q_{\mathrm{PAH}}$ across the galaxy by fitting the SED models of Draine \& Li (2007) at every independent pixel in the map. Details of the SED modeling are described in Sandstrom et al. (2010) along with a more thorough description of the results. In addition to modeling the SED, we incorporate the $\mathrm{S}^{4} \mathrm{MC}$ spectroscopy in the overlap regions to test whether the photometric determination of $q_{\mathrm{PAH}}$ does an adequate job of judging the PAH fraction, with essentially only a measurement of the $8 \mu \mathrm{m}$ feature. Figure 1 shows the best fit models to the photometry only and combined photometry and spectroscopy for a few locations in our maps. We find that the photometric only determination of $q_{\mathrm{PAH}}$ is very close to the best fit $q_{\mathrm{PAH}}$ from the combined fit for all of the overlap regions.

In Figure 2 we show the PAH fraction map that results from our SED fits. We find that the SMC has an average $q_{\mathrm{PAH}}=0.6 \%$, significantly below the Milky Way value of $q_{\mathrm{PAH}, \mathrm{MW}}=4.6 \%$. There are large spatial variations in the SMC PAH 

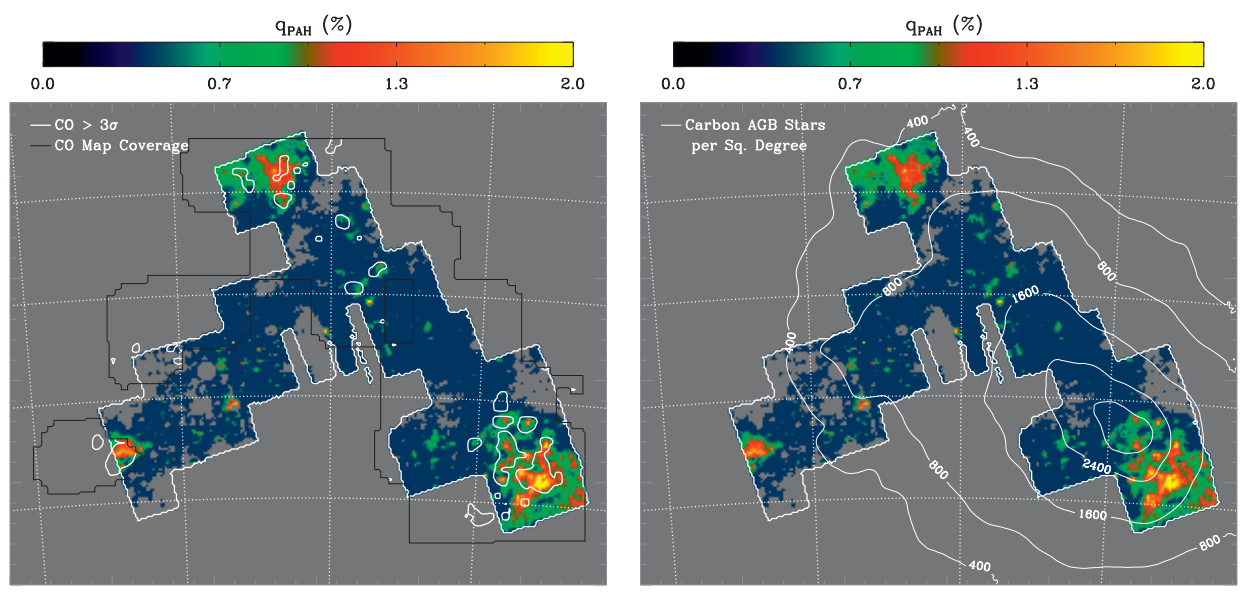

Fig. 2. The PAH fraction maps that result from our SED fitting overlaid with contours of $\mathrm{CO} \mathrm{J}=(1-0)$ from the NANTEN survey (left) and carbon star surface density (right). It is clear that the PAH fraction is correlated with the locations where molecular gas is present but not with the distribution of evolved stars.

abundance. At maximum $q_{\mathrm{PAH}}$ can be within a factor of two of the Milky Way level, but most of the galaxy has $q_{\mathrm{PAH}}=0.4 \%$, which represents the lower limit of our models. For its metallicity, the SMC average $q_{\mathrm{PAH}}$ agrees well with the average $q_{\mathrm{PAH}} \sim 1 \%$ found by Draine et al. (2007) for the SINGS sample.

Next, we compare the PAH fraction to various proposed drivers for the PAH abundance. Evolved stars are thought to be the dominant source of PAHs. We trace the distribution of evolved stars using color selection criteria on the 2MASS catalog to select carbon AGB stars (Cioni et al. 2006). The distribution of carbon stars follows the well known spheroidally distributed population of old stars in the SMC (Harris \& Zaritsky 2004). There is no correspondence between the evolved star density and the PAH fraction, in contrast to what is seen in the LMC, where the PAH fraction is seen to be enhanced along the stellar bar (Paradis et al. 2009). We find that the PAH fraction is correlated with the presence of molecular gas as traced by $\mathrm{CO} J=(1-0)$ from the NANTEN survey (Mizuno et al. 2001). Since the molecular clouds condense out of the diffuse ISM on timescales of $\sim 25 \mathrm{Myr}$ (Fukui et al. 1999), there must be some process which allows the molecular clouds and the diffuse ISM they condensed out of to have different PAH fractions. Two possibilities are: 1) a recent $(<25 \mathrm{Myr})$ event which destroyed PAHs in the diffuse ISM over the whole galaxy after the current generation of molecular clouds condensed; or 2) some component of interstellar PAH material forms in molecular clouds. The SMC star-formation history shows little evidence for a recent event that could have altered the $\mathrm{PAH}$ fraction globally, so we consider the latter option more likely. 


\section{The physical state of PAHs in the SMC}

The ratios of the mid-IR PAH bands provide a wealth of information about the physical state of PAHs-charge, size, structure and composition all alter the relative strengths of the mid-IR features. Understanding the physical state of PAHs in the SMC may help us to explain the low-metallicity PAH deficit. For instance, PAHs that are smaller or ionized are easier to photodissociate under typical ISM conditions (Allain et al. 1996a, 1996b). To study the physical state of PAHs in the SMC, we have used the $\mathrm{S}^{4} \mathrm{MC}$ spectroscopy and measured the strengths of the various bands. A detailed description of this work can be found in Sandstrom et al. (2010, in prep.).

In Figure 3 we show measurements of the band ratios overlaid on theoretical predictions from Li \& Draine (2001). The gray lines show neutral (top) and singly-ionized (bottom) PAHs with a variety of sizes (labeled on the bottom plot). Since interstellar PAHs come with a size and charge distribution, the theoretical predictions are only used as a guide for interpreting the ratios relative to other measurements. We also plot the band ratios measured in the SINGS galaxies (without AGN; Smith et al. 2007). The SINGS spectroscopy covers regions with approximately solar metallicity. Compared to SINGS, we find that the SMC PAHs tend to be more neutral and smaller, judging from the ratios of their $6-12 \mu \mathrm{m}$ features. In addition, we find that the $17 \mu \mathrm{m}$ PAH complex is notably weak in the SMC, continuing a trend noted by Smith et al. (2007) for the strength of the 17 feature to decrease as a function of metallicity.

\section{Insights into the PAH life cycle}

To summarize, the Spitzer surveys of the SMC ( $\mathrm{S}^{3} \mathrm{MC}$ and $\left.\mathrm{S}^{4} \mathrm{MC}\right)$ allow us to study the abundance and physical state of PAHs in a nearby, low-metallicity galaxy. We find that the SMC PAH fraction is significantly below the Milky Way value, but with large spatial variations. High PAH fractions are found in molecular regions, while the diffuse ISM is essentially devoid of PAHs (to the limit of our models). Spectroscopic investigations of a number of the main star-forming regions show that the mid-IR band ratios suggest smaller and more neutral PAHs than found in more metal rich galaxies (for example in the SINGS sample).

These results suggest a scenario where PAH formation in dense regions is important, at least at low-metallicity. The process by which this proceeds is currently uncharacterized, but may depend on the coagulation of material on the surfaces of existing grains. The PAHs that result from this formation channel in the SMC seem to be smaller on average than PAHs in more metal rich galaxies, judging by their band ratios. Smaller PAHs are easier to photodissociate under typical diffuse ISM conditions, perhaps leading to the very low PAH abundance over most of the SMC. PAHs formed in the atmospheres of evolved stars are mainly injected first into the diffuse ISM and may be largely destroyed in the SMC. At higher metallicity, we speculate that PAHs are better able survive in the diffuse ISM, possibly because of softer and less intense UV fields and/or larger average PAH sizes. As 


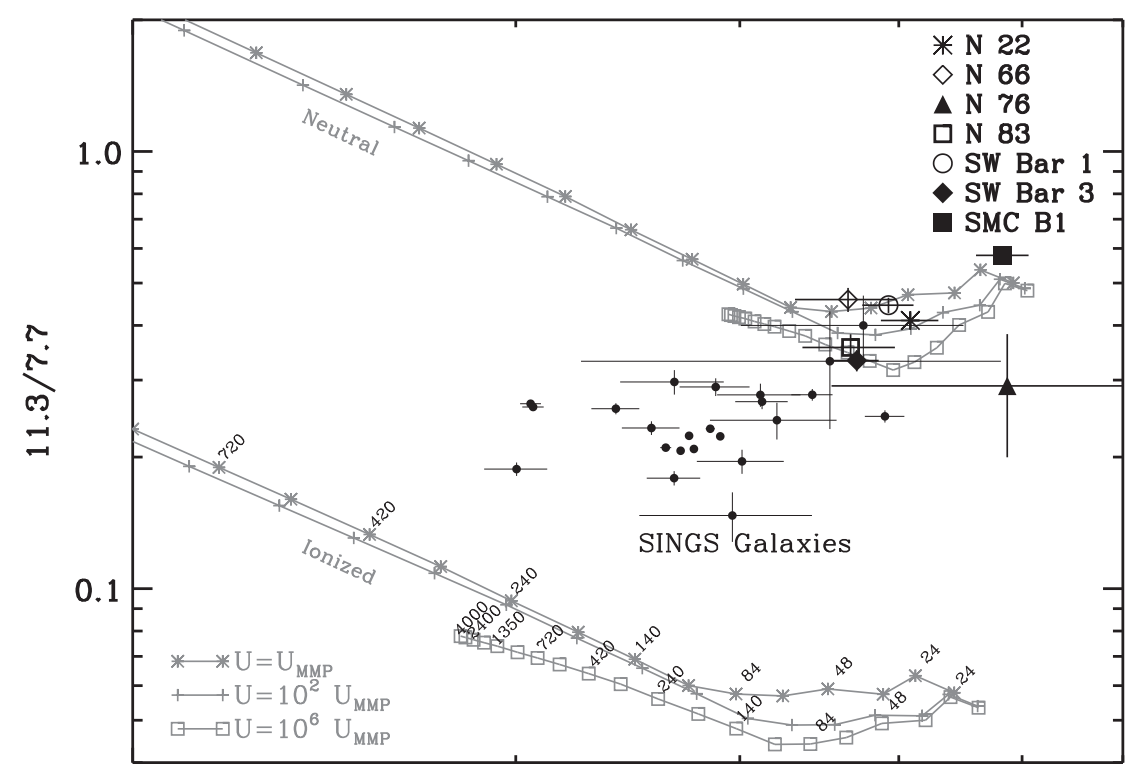

0.1

$6.2 / 7.7$

Fig. 3. Ratios of the 6.2, 7.7 and $11.3 \mu \mathrm{m}$ PAH bands in the SMC compared with the SINGS sample and the theoretical models of Li \& Draine 2001. The band ratios suggest that SMC PAHs are smaller and more neutral than PAHs in the SINGS galaxies.

PAHs become able to survive in the diffuse ISM, the overall PAH fraction of a galaxy can build up to Milky Way levels. Resolved studies of the PAH fraction in a larger sample of nearby galaxies that span a range of metallicity will allow us to study these trends.

\section{References}

Allain, T., Leach, S., \& Sedlmayr, E., 1996a, A\&A, 305, 602

Allain, T., Leach, S., \& Sedlmayr, E., 1996b, A\&A, 305, 616

Bolatto, A.D., Simon, J.D., Stanimirović, S., et al., 2007, ApJ, 655, 212

Cherchneff, I., Barker, J.R., \& Tielens, A.G.G.M., 1992, ApJ, 401, 269

Cioni, M.-R.L., Girardi, L., Marigo, P., \& Habing, H.J., 2006, A\&A, 452, 195

Draine, B.T., 2009, in Astronomical Society of the Pacific Conf. Ser., 414, ed. T. Henning, E. Grün, \& J. Steinacker, 453

Draine, B.T., \& Li, A. 2007, ApJ, 657, 810

Draine, B.T., Dale, D.A., Bendo, G., et al., 2007, ApJ, 663, 866

Engelbracht, C.W., Gordon, K.D., Rieke, G.H., et al., 2005, ApJ, 628, L29

Engelbracht, C.W., Rieke, G.H., Gordon, K.D., et al., 2008, ApJ, 678, 804 
Fukui, Y., Mizuno, N., Yamaguchi, R., et al., 1999, PASJ, 51, 745

Galliano, F., Dwek, E., \& Chanial, P., 2008, ApJ, 672, 214

Gordon, K.D., Engelbracht, C.W., Rieke, G.H., et al., 2008, ApJ, 682, 336

Harris, J., \& Zaritsky, D., 2004, AJ, 127, 1531

Latter, W.B., 1991, ApJ, 377, 187

Li, A., \& Draine, B.T., 2001, ApJ, 554, 778

Madden, S.C., 2000, New Astron. Rev., 44, 249

Marble, A.R., Engelbracht, C.W., van Zee, L., et al., 2010, ApJ, 715, 506

Matsuura, M., Barlow, M.J., Zijlstra, A.A., et al., 2009, MNRAS, 396, 918

Micelotta, E.R., Jones, A.P., \& Tielens, A.G.G.M., 2010, A\&A, 510, A36

Miville-Deschênes, M.-A., Boulanger, F., Joncas, G., \& Falgarone, E., 2002, A\&A, 381, 209

Mizuno, N., Rubio, M., Mizuno, A., et al., 2001, PASJ, 53, L45

O'Halloran, B., Satyapal, S., \& Dudik, R.P., 2006, ApJ, 641, 795

Paradis, D., Reach, W.T., Bernard, J.-P., et al., 2009, AJ, 138, 196

Sandstrom, K.M., Bolatto, A.D., Draine, B.T., Bot, C., \& Stanimirović, S., 2010, ApJ, 715,701

Sandstrom, K.M., Bolatto, A.D., Stanimirović, S., van Loon, J.T., \& Smith, J.D.T., 2009, ApJ, 696, 2138

Smith, J.D.T., Draine, B.T., Dale, D.A., et al., 2007, ApJ, 656, 770

Wu, Y., Charmandaris, V., Hao, L., et al., 2006, ApJ, 639, 157 
